\title{
Dipolar Doping of Organic Semiconductors to Enhance Carrier Injection
}

\author{
Alexander J.L. Hofmann $\odot,{ }^{1},{ }^{*}$ Simon Züfle $\odot,{ }^{2,3}$ Kohei Shimizu $\odot,{ }^{4}$ Markus Schmid, ${ }^{1}$ Vivien Wessels, ${ }^{1}$ \\ Lars Jäger, ${ }^{1}$ Stéphane Altazin, ${ }^{3}$ Keitaro Ikegami ${ }^{4}$ Motiur Rahman Khan $\odot,{ }^{5}$ Dieter Neher, ${ }^{5}$ \\ Hisao Ishii $\oplus^{4,6,7}$ Beat Ruhstaller, ${ }^{2,3}$ and Wolfgang Brütting ${ }^{1, \dagger}$ \\ ${ }^{1}$ Institute of Physics, University of Augsburg, 86135 Augsburg, Germany \\ ${ }^{2}$ Institute of Computational Physics, ZHAW, 8401 Winterthur, Switzerland \\ ${ }^{3}$ Fluxim AG, 8400 Winterthur, Switzerland \\ ${ }^{4}$ Graduate School of Science and Engineering, Chiba University, Chiba 263-8522, Japan \\ ${ }^{5}$ Physik weicher Materie, University of Potsdam, 14476 Potsdam, Germany \\ ${ }^{6}$ Center for Frontier Science, Chiba University, Chiba 263-8522, Japan \\ ${ }^{7}$ Molecular Chirality Research Center, Chiba University, Chiba 263-8522, Japan
}

(Received 28 May 2019; revised manuscript received 7 November 2019; published 24 December 2019)

If not oriented perfectly isotropically, the strong dipole moment of polar organic semiconductor materials such as tris-(8-hydroxyquinolate)aluminum $\left(\mathrm{Alq}_{3}\right)$ will lead to the buildup of a giant surface potential (GSP) and thus to a macroscopic dielectric polarization of the organic film. Despite this having been a known fact for years, the implications of such high potentials within an organic layer stack have only been studied recently. In this work, the influence of the GSP on hole injection into organic layers is investigated. Therefore, we apply a concept called dipolar doping to devices consisting of the prototypical organic materials $N, N^{\prime}$-Di(1-naphthyl)- $N, N^{\prime}$-diphenyl-(1,1'-biphenyl)-4,4'-diamine (NPB) as nonpolar host and $\mathrm{Alq}_{3}$ as dipolar dopant with different mixing ratios to tune the GSP. The mixtures are investigated in single-layer monopolar devices as well as bilayer metal/insulator/semiconductor structures. Characterization is done electrically using current-voltage $(I-V)$ characteristics, impedance spectroscopy, and charge extraction by linearly increasing voltage and time of flight, as well as with ultraviolet photoelectron spectroscopy. We find a maximum in device performance for moderate to low doping concentrations of the polar species in the host. The observed behavior can be described on the basis of the Schottky effect for image-force barrier lowering, if the changes in the interface dipole, the carrier mobility, and the GSP induced by dipolar doping are taken into account.

DOI: 10.1103/PhysRevApplied.12.064052

\section{INTRODUCTION}

Since the introduction of multilayer devices in the mid-1990s, organic semiconductors have emerged as a widely used material set for state-of-the art lighting and display applications, where organic light-emitting diodes (OLEDs) provide high image quality and organic photovoltaics (OPV) have passed the first hurdle in progress toward commercialization. Still, many aspects of organic electronics are not fully understood or in turn offer scope for optimization. For OLEDs, the orientation of the transition dipole moment of the emitter molecules, as well as its influence on device performance [1], has been extensively studied in the past $[2,3]$. However, many of the molecules used in organic electronics also have a permanent dipole

\footnotetext{
*alexander.hofmann@physik.uni-augsburg.de; http://www.physik.uni-augsburg.de/organic

${ }^{\dagger}$ wolfgang.bruetting@physik.uni-augsburg.de
}

moment that, if not oriented perfectly isotropically, will lead to the buildup of a giant surface potential (GSP) due to spontaneous orientation polarization (SOP) and thus to a macroscopic dielectric polarization of the organic film [4]. Besides the physics behind the orientation mechanism upon film growth and the relation between the orientation of both the permanent and the transitional dipole moment [3], the influence of the GSP on OLED device performance is also still under investigation [5].

An organic light-emitting diode, in which at least one of the layers shows this dielectric polarization, is herein considered a "polar OLED." In this work, we focus on the effect of SOP on device performance in polar OLEDs, especially on carrier injection into the active layers. One of the most important effects of the dipolar nature of the respective layer is that it induces a change in electric field distribution provoked by the buildup of interface charges, which would otherwise be determined mostly by the contact potentials and the 
applied voltage [4]. Previously, the effect of dipole-induced interface charges on electron injection from the cathode into the electron transport layer (ETL) of such a polar OLED has been investigated by Noguchi et al. by comparing devices with $\mathrm{Alq}_{3}$ and $\mathrm{Al}(7-\mathrm{Prq})_{3}$ (tris(7-propyl-8hydroxyquinolinato aluminum) as ETL [6], where electron injection was improved or hindered, respectively. Later on, this device structure was described by driftdiffusion simulation by Altazin et al. [5] and a possible explanation for the effect was given by Kinjo et al. [7]. The main difference between those two materials is the sign of their overall orientation polarization and hence the sign of the interface charge at the ETL-cathode interface [6].

To study the influence of a surface potential on hole injection at the anode side, OLEDs incorporating a polar hole-transport layer (HTL), preferably with a tunable magnitude of the GSP, are needed. While many common electron-transporting materials are indeed polar with a nonzero permanent dipole moment $[8,9]$ and also show a GSP in vacuum-evaporated organic films due to SOP, most available hole-conducting materials are either nonpolar or show isotropic orientation.

Therefore, in this work, a nonpolar hole-transporting host is diluted or doped by polar guest molecules. In a previous work, we have shown that the overall GSP of the ETL in an OLED can be tuned by diluting the polar ETL by a nonpolar material [10]. We found that, for low doping ratios below $10 \%$, the polar dopant shows almost ideal alignment of its dipole moments. This concept is herein reversed to dipolar doping of $\mathrm{Alq}_{3}$ into NPB to achieve a varying GSP, while avoiding changing the hole-transport material itself as much as possible. For this purpose, we choose the prototypical organic semiconductors NPB in conjunction with $\mathrm{Alq}_{3}$ as the base material set for our studies. Additionally, to investigate the GSP effect on hole injection, we vary the work function of the anode. Besides the presumed positive effect on carrier injection due to the interface charges, doping and dipolar disorder [11] might negatively influence the charge-carrier transport of the host and thus will lead to a competition between various effects that have to be accounted for.

To determine the magnitude of the hole-injection barrier from a working device, we apply our recently published method, utilizing impedance spectroscopy (IS) and charge extraction by linearly increasing voltage (CELIV) on metal-insulator-semiconductor (MIS) diodes; see Ref. [12]. Additionally, hole-only single carrier devices with only the doped HTL material are measured. For comparison, hole mobilities are determined using time of flight (TOF) with various doping concentrations. A selected set of samples is also cross-checked using ultraviolet-photoelectron spectroscopy (UPS).

\section{EXPERIMENTAL}

For electrical measurements, devices with active areas of 0.9 and $4 \mathrm{~mm}^{2}$ are fabricated in our laboratory. Substrates are cleaned and pretreated with UV and ozone prior to spin-coating or nitrogen plasma when they are to be used directly, without an additional hole-injection layer. Polymeric poly(3,4-ethyl enedioxythiophene):poly(styrene-sulfonate) (PEDOT:PSS) derivatives are spin-coated in a clean room, while organic small-molecule materials and metals are thermally evaporated under high-vacuum conditions $(p \approx$ $10^{-7}$ mbar). Thicknesses are monitored in situ with quartzcrystal microbalances and cross-checked ex situ using a Veeco/Bruker Dektak 8 profilometer with the sample covered under a $100-\mathrm{nm}$ protective gold layer. Samples can either be measured directly in a glove box with a nitrogen atmosphere or transferred in vacuum between chambers. The areas of some samples are cross-checked using optical microscopy after the electrical measurements are done.

Two main layer stacks are investigated in this work, for mono- and bipolar devices, respectively. The layer stack for monopolar devices is ITO/(HIL)/NPB:Alq $3 / \mathrm{HATCN} /$ $\mathrm{Au}$ and for impedance and CELIV measurements (bipolar) it is $\mathrm{ITO} /(\mathrm{HIL}) / \mathrm{NPB}: \mathrm{Alq}_{3} / \mathrm{Alq}_{3} / \mathrm{Ca} / \mathrm{Al}$, where "ITO" refers to the transparent conductive indium tin oxide commonly used in organic semiconductor devices. A sketch of an energy-level diagram with a layer structure is given in Fig. 1 for both sample types. For both monopolar and bipolar devices, the hole-injecting contact or anode can either
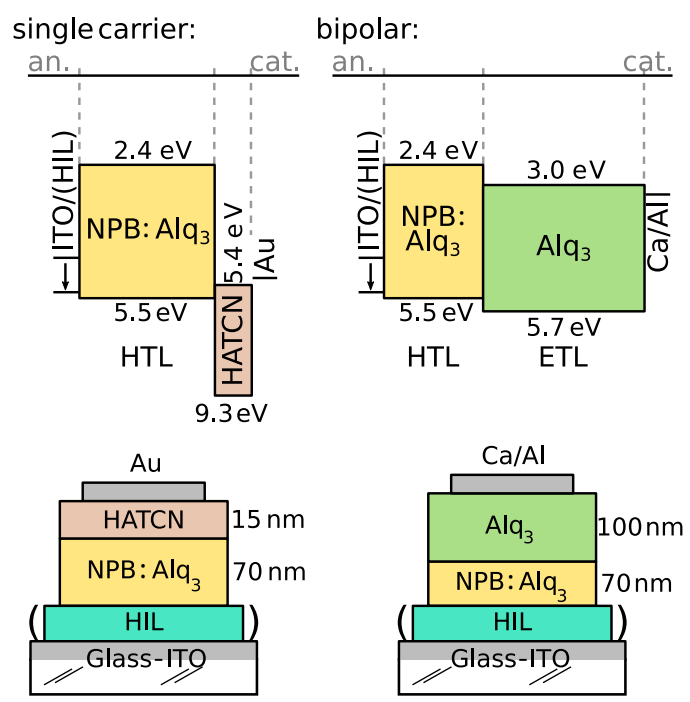

FIG. 1. Top: a sketch of the energy levels for both device types under investigation. The top and bottom electrodes (cathode and anode, respectively) are marked. The substrate work function is changed between approximately 4.6 and $5.7 \mathrm{eV}$. The energy levels are taken from Refs. [13] and [4] or measured in our groups using UPS and IPES. Bottom: a schematic of the stack layout for both types of samples. The HIL layer is omitted for samples processed directly on ITO. 
be bare ITO or ITO covered with the commercially available PEDOT:PSS formulation Clevios HIL1.3 (designated as HIL). The focus of this study lies on the anode contact at the bottom of the device. The mixing ratio of $\mathrm{Alq}_{3}$ in NPB, referred to in the following as the doping ratio, is varied between $0 \%$ (neat NPB) and about $10 \%$ of $\mathrm{Alq}_{3}$ in NPB; the value refers to the volume percentage calculated from the individual thicknesses of $\mathrm{Alq}_{3}$ and NPB as measured during evaporation. The molar doping ratio is slightly higher due to different densities in neat and mixed systems; however, the overall difference is relatively small.

Samples for TOF feature a 200-nm-thick transport layer of doped NPB on unstructured ITO topped with a thin (approximately $20-\mathrm{nm}$ ) charge-generation layer of $\mathrm{C}_{70}$ followed by bathocuproine (BCP, $5 \mathrm{~nm}$ ) and Al. Measurements are carried out by exciting the samples with a wavelength-tunable diode-pumped $Q$-switched Nd:YAG laser (NT242, EKSPLA) with an 8-ns pulse-duration time at an excitation wavelength of $532 \mathrm{~nm}$ and $500-\mathrm{Hz}$ repetition frequency. A $50-\Omega$ resistor is used to measure the current, which is recorded using an Agilent Infiniium DSO9054H oscilloscope.

The $I-V$ characterization, as well as impedance spectroscopy (IS) and charge extraction by linearly increasing voltage (CELIV) are conducted using the PAIOS characterization platform [14].

Samples for ultraviolet photoelectron spectroscopy (UPS) feature an incomplete layer stack of ITO/NPB:Alq 3 to measure the contact barrier between ITO and the HTL. Measurements are conducted using low-energy UPS [15] with excitation energies between 4.4 and $21.22 \mathrm{eV}$ to accurately determine the barriers.

\section{RESULTS}

\section{A. Barrier-dependent $I-V$ characteristics on monopolar devices}

To investigate carrier transport within dipolar doped NPB, single carrier devices with only the hole-transport layer are fabricated and compared. When electron injection is effectively blocked from both contacts, the current through the device is dependent on the bulk-transport properties as well as the injection of holes only. If one contact is held constant while the injection properties are altered at the other one by changing the anode material and work function, the difference in current can be a measure of the hole-injection barrier at the anode.

The hole-injecting material for the bottom anode is chosen from either ITO with $\Phi_{\text {ITO }} \approx 4.6 \mathrm{eV}$ or HIL1.3 with $\Phi_{\text {HIL1.3 }}=5.7 \mathrm{eV}$ [13]. For the top contact, i.e. the cathode, a sequence of HATCN and gold is evaporated on the device, which is reported to show very low barriers [16] and might hence serve as a reference for hole injection compared to the anode.

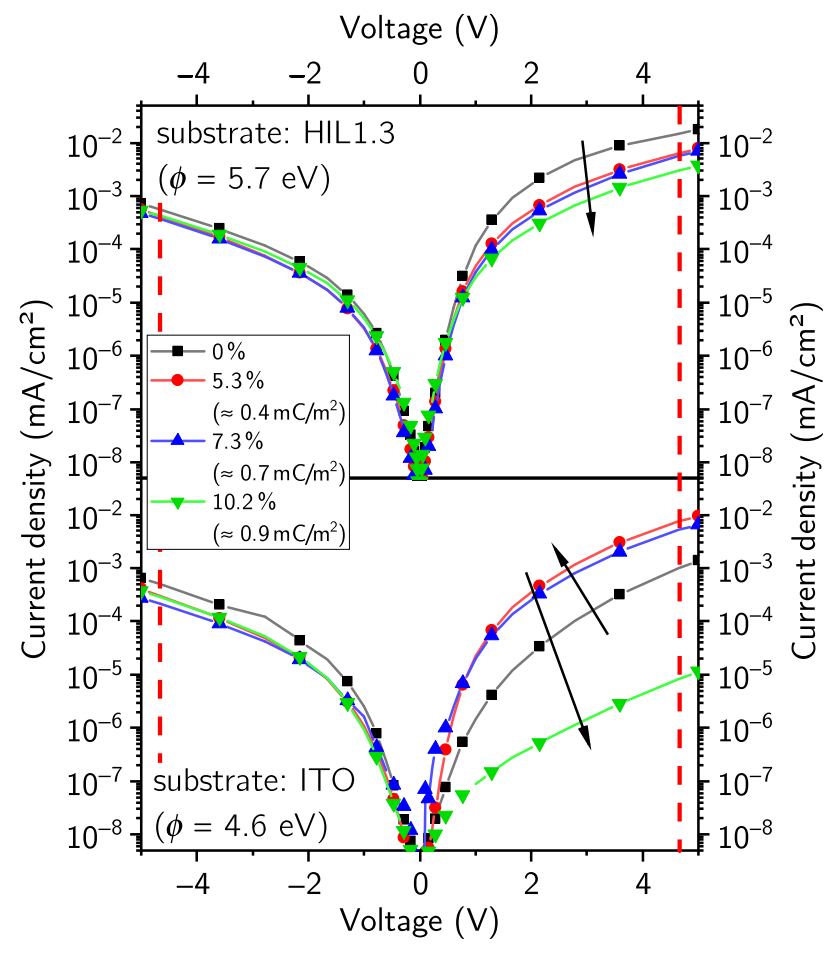

FIG. 2. Measurements on NPB:Alq 3 mixtures in hole-only devices. The contacts are as such that the current on the left side or with negative voltage resembles injection through the HATCN-gold interface, where the positive voltage range (the right-hand side) shows injection over the anode-NPB:Alq ${ }_{3}$ interface. The anode materials used for each device are noted on the graph, together with their work functions.

For both anode materials, different doping ratios for $\mathrm{Alq}_{3}$ in NPB are processed and measured, with an interface charge density ranging from $0 \mathrm{mC} \mathrm{m}^{-2}$ to roughly 1 $\mathrm{mC} \mathrm{m}^{-2}$ as calculated from impedance spectroscopy measurements on bipolar diodes with the same doping ratio (see Sect. III B for details).

The $I-V$ characteristics are shown in Fig. 2. The normal undoped NPB hole-only device with no GSP is shown in black (square data points). Here, the hole current is almost one order of magnitude higher with HIL on the anode compared to bare ITO, which can be related to the high substrate work function of HIL1.3 and the corresponding low-hole-injection barrier. For HIL, rising doping ratios lead to a steady decrease of the current by almost one order of magnitude. In the case of a low work function or high-barrier hole injection through ITO on the anode-NPB:Alq 3 side, however, moderate doping of roughly $5 \%$ leads to the highest currents in the device. For a better comparison and to further investigate those findings, a figure of merit describing the "injection quality" of the anode-side contact is introduced. The contact through HATCN/Au is reproducibly good, with only a slight influence on the current; we therefore take this side as a reference and plot the logarithm of the ratio of the 


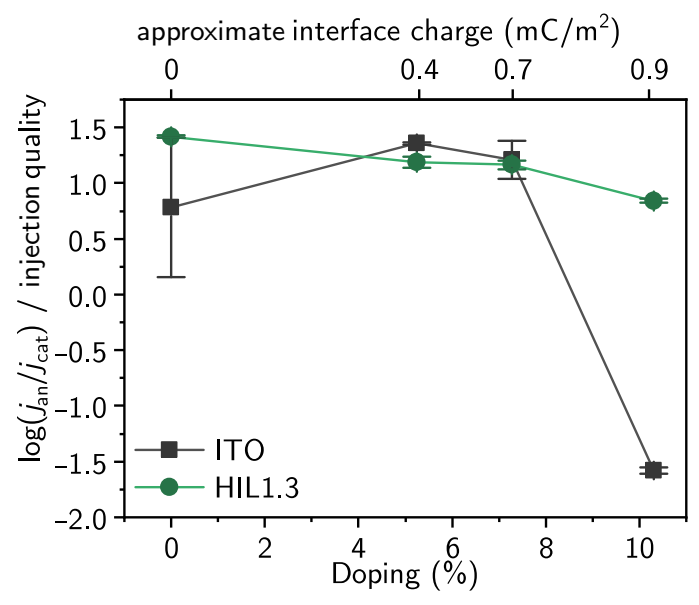

FIG. 3. The logarithm of the forward-to-reverse current ratio evaluated at $4.65 \mathrm{~V}$. Higher values therefore represent an improved contact at the anode, just as a rising trend indicates improving injection.

current injected through the HATCN/Au cathode and that through the ITO/(HIL) anode taken at $\pm 4.65 \mathrm{~V}$ against the doping ratio in Fig. 3, the position of this evaluation being marked in Fig. 2 with vertical lines. In this evaluation, positive values indicate a higher current and hence presumably better hole injection though the anode side, while negative values state the opposite. Likewise, a positive slope of the plot indicates improving - and a negative slope impaired-injection through the anode-side interface. As a side effect, by division, the effects of changes in bulk charge transport due to changed mobility cancel each other out, as they appear on both sides equally. In turn, of course, mobility-dependent effects at the contacts, such as space-charge or diffusion-limited injection [17], will still be visible. Note that the description of carrier injection through this HATCN layer is nontrivial [18] and the influence of the polar nature of the active layer on carrier injection through HATCN is also unknown and not the subject of this work.

At first, the case of pure ITO as the hole-injecting contact is considered. For low doping of up to $5 \%-7 \%$, the measurement shows a higher current or better injection for a rising $\mathrm{Alq}_{3}$ concentration, leading to a positive trend in the injection-quality plot. When the doping ratio is further increased, the graph does not show any saturation; instead, the injected current decreases again, as indicated by a negative trend in the injection-quality graph. The second case, with HIL at the anode, shows a negative trend over all different doping ratios. Interestingly, the current injected through the HIL is clearly higher by approximately one order of magnitude compared to the HATCN/Au contact, as is directly evident due to positive values in the plot. The HIL samples also outperform the ITO stack at $0 \%$ and $10 \%$ doping, but not at the optimal doping ratio around 5\%.
Assuming that the GSP of the HTL has a direct influence on the injection barrier between the anode and the HTL, the observations made with HIL meet our expectations regarding the high-work-function substrate. When the presumed barrier into NPB is already low, which is the case for HIL with $\Phi_{\mathrm{HIL} 1.3} \approx 5.7 \mathrm{eV}$ [13], the effective barrier is subject to Fermi-level pinning: Greiner et al. have shown that the resulting injection barrier saturates at a finite nonzero value, even if the substrate work function is near to or larger than the organic's ionization potential (IP) [19]. It is therefore unlikely that any improvement of injection due to GSP is possible, considering the IP of NPB at $5.3 \mathrm{eV}$. Instead, the measurement in Fig. 2 shows a small negative trend, indicating impaired injection through the HIL/HTL interface. By contrast, with an ITO work function of roughly $4.6 \mathrm{eV}$, a nominal injection barrier of up to $1 \mathrm{eV}$ can be expected in the first case, which leaves room for improvement. Up to roughly $5 \%$, the current is indeed positively influenced, as expected from comparison with Altazin and coworkers [5]. The decreased current through the ITO-NPB:Alq ${ }_{3}$ interface for higher doping ratios, however, is surprising, not least because it differs by more than two orders of magnitude from the undoped case. From the calculation of the aforementioned figure of merit, we can conclude that a change in bulk carrier transport of the NPB:Alq ${ }_{3}$ mixture alone cannot be the root cause of the observed optimum, as its influence would affect both the forward and reverse sides of the $I-V$ characteristics equally. Hence, interface-bound effects such as changes in the barrier due to, e.g., altered energy levels of NPB and/or mobility-limited carrier injection, have to be accounted for. To further investigate the anode interface, polar MIS diodes are fabricated.

\section{B. Bilayer MIS structures}

Recently, we have published a method utilizing temperature-dependent impedance spectroscopy combined with CELIV measurements to extract both carrier mobility and mobility activation as well as the injection barrier from the same device. For a detailed description, we refer to the work of Züfle et al. [20]: a short description of the technique is as follows. In polar OLEDs, the GSP of the ETL causes a change in the electric field distribution that eventually leads to an accumulation of charge carriers - holes in our case - in the nonpolar HTL at the interface between these two adjacent layers [4]. Above a specific transition voltage $V_{\text {tr }}$, these accumulated charges increase the respective layer's conductivity such that its former contribution to the overall (geometric) capacitance response of the device vanishes and only the ETL capacitance is obtained. As we have shown, the same concept applies to doped layers [3,10]; also, the interface charges of both layers are additive [8] in two-layer systems. 
(a)

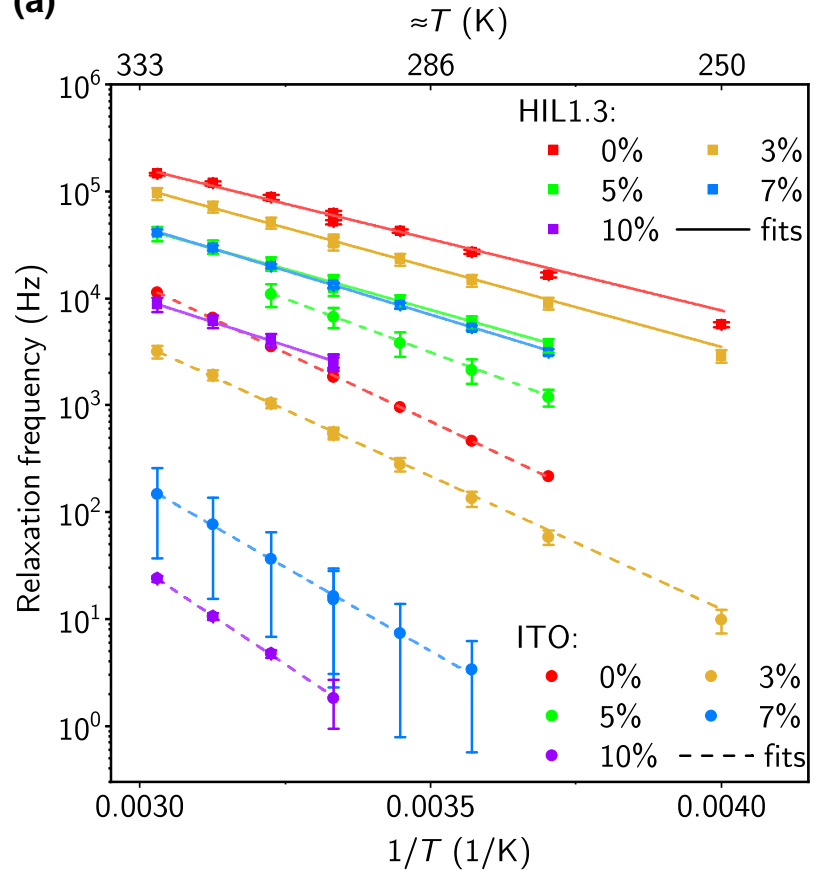

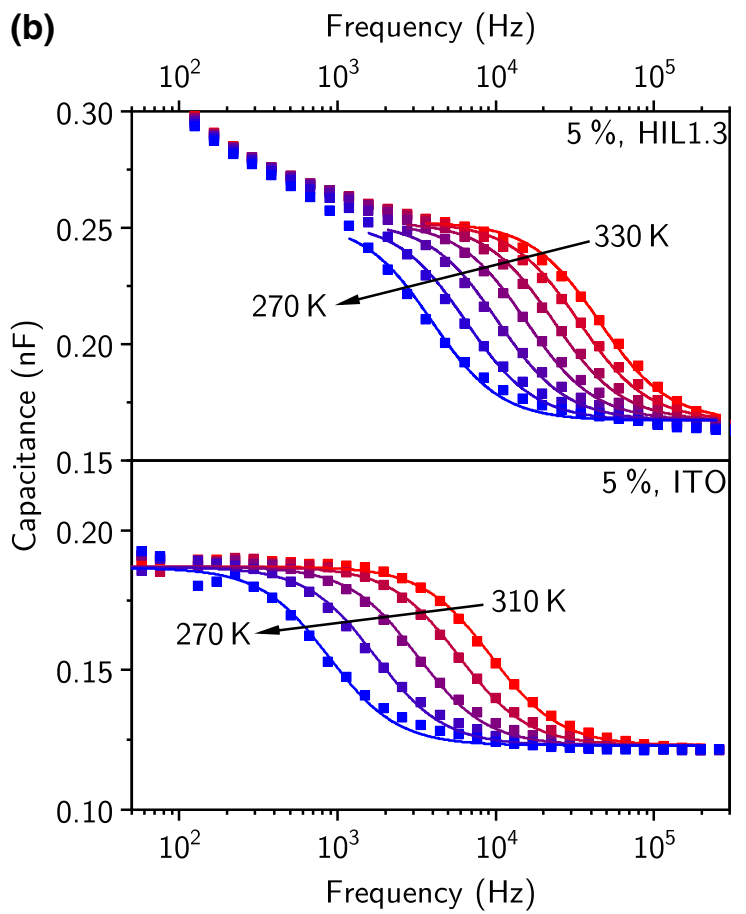

FIG. 4. (a) Measurements of the temperature dependence of the relaxation frequency for different doping for ITO- (dashed-line fits) and HIL- (straight-line fits) based devices. The points and squares denote the measurements at various temperatures and the lines are the fits. The temperature is varied between 330 and $250 \mathrm{~K}$, depending on the stability of the respective samples and on contacting. (b) Examples of $C-f$ measurements (see the text); here, for $5 \%$ of doping. The ITO-based sample degrades above $310 \mathrm{~K}$ and the measurements at 320 and $330 \mathrm{~K}$ are hence not shown. The straight lines connecting the data points are fits based on a simple 2-RC model.

When measuring the capacitance response of the device while varying the frequency ( $C-f$ measurement) at a constant dc bias voltage between $V_{\text {tr }}$ and the built-in voltage $V_{\mathrm{bi}}$, the transition from the geometric capacitance to the ETL capacitance (the accumulation regime) is observed at a characteristic frequency commonly referred to as the relaxation frequency $f_{\text {rel }}$. The temperature dependence of this relaxation frequency is known to be a measure of the thermal activation of the hole current in the device [21]. We relate it to injection barriers and bulk-mobility activation [20]. It is commonly obtained from an Arrhenius analysis by assuming a simple exponential dependence:

$$
f_{\text {rel }}=f_{0} \exp \left(\frac{-E_{\text {act }}}{\mathrm{k}_{B} T}\right)=f_{0} \exp \left(\frac{-E_{\mu}-E_{\text {inj }}}{\mathrm{k}_{B} T}\right),
$$

where the total activation energy $E_{\text {act }}$ can be written as $E_{\text {act }}=E_{\mu}+E_{\text {inj }}$, with the mobility activation $E_{\mu}$ and the injection barrier $E_{\text {inj. }}$. With polar MIS-CELIV, the mobility and its activation can be determined on the same devices, thus allowing us to disentangle hole injection into and transport through the HTL $[12,20]$.

In this work, temperature-dependent measurements are conducted on bilayer devices with doping ratios between $0 \%$ and $10 \% \mathrm{Alq}_{3}$ in NPB. Additionally, bare ITO and
HIL allow us to compare different electrode work functions and nominal injection barriers, respectively. Example measurements and fits to determine relaxation frequencies are shown in Fig. 4. The extracted absolute relaxation frequencies are also given in an Arrhenius plot, together with fits used to determine the activation energies. On bare ITO, the larger injection barrier leads to considerably higher resistivity and hence orders-of-magnitudelower relaxation frequencies compared to samples with HIL as the hole-injection layer. For ITO, the highest relaxation frequencies are observed for moderate doping ratios (dashed lines in Fig. 4), whereas samples with HIL on the anode follow a steady decay in relaxation frequency with the doping ratio (solid lines). Also, it is already clearly visible to the naked eye that the slopes of the fits differ more for the ITO-based samples, with the 5\% mixture showing the smallest slope, together with the highest relaxation frequency among this series. This is consistent with the findings from the $I-V$ characteristics. From the slopes of the temperature-dependent measurements, we obtain the activation energies for charge-carrier injection. Figure 5 shows the extracted activation energies calculated by fitting Eq. (1) onto the temperature-dependent $C-f$ measurements, as well as the mobility activation obtained from the temperature-dependent CELIV mobilities. The 


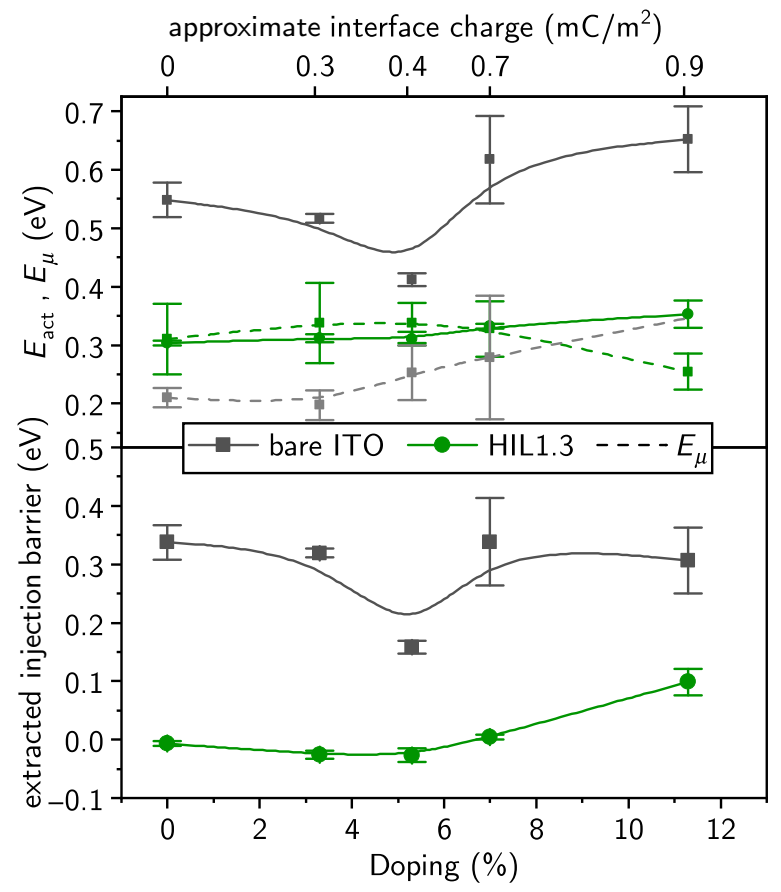

FIG. 5. Extracted parameters from impedance spectroscopy and MIS-CELIV for different doping and barriers. The work functions of the different anode layers are around $4.6 \mathrm{eV}$ for bare ITO and $5.7 \mathrm{eV}$ in the case of HIL. As a guide to the eye, samples with the same anode material are connected with a spline. The top portion shows the measurement quantities, while the solid lines refer to the frequency activation and the dashed lines to the mobility activation. The bottom portion shows the calculated injection barrier.

injection barrier $E_{\mathrm{inj}}=E_{\text {act }}-E_{\mu}$ is also given in the lower part.

Subtracting the mobility activation from the measured overall frequency activation yields the injection barrier depicted in the bottom part of the graph. In the case of a high-work-function substrate such as HIL with $\Phi_{\text {HIL } 1.3} \approx$ $5.7 \mathrm{eV}$ [13], the presumed effective barrier for hole injection into NPB is already low and is additionally affected by Fermi-level pinning [19], as also seen in the $I-V$ characteristics. The method used to determine the barriers is known to underestimate the extracted value for injection barriers below $300 \mathrm{meV}$ [12], which can also be seen here with values around zero. Still, the overall picture shows a very small barrier that is not further lowered by the increasing interfacial charge density.

For bare ITO with $\Phi_{\text {ITO }} \approx 4.6 \mathrm{eV}$, however, a lowering of the barrier with increasing interfacial charge density is possible and indeed observed for moderate doping of up to $5 \%$. Given that the subtraction of the mobility activation already accounts for the mobility effect at the interface in the case of impedance spectroscopy, the rise in the barrier for higher dilutions must be attributed to different processes at the ITO-NPB:Alq 3 interface or in the bulk of the doped NPB.

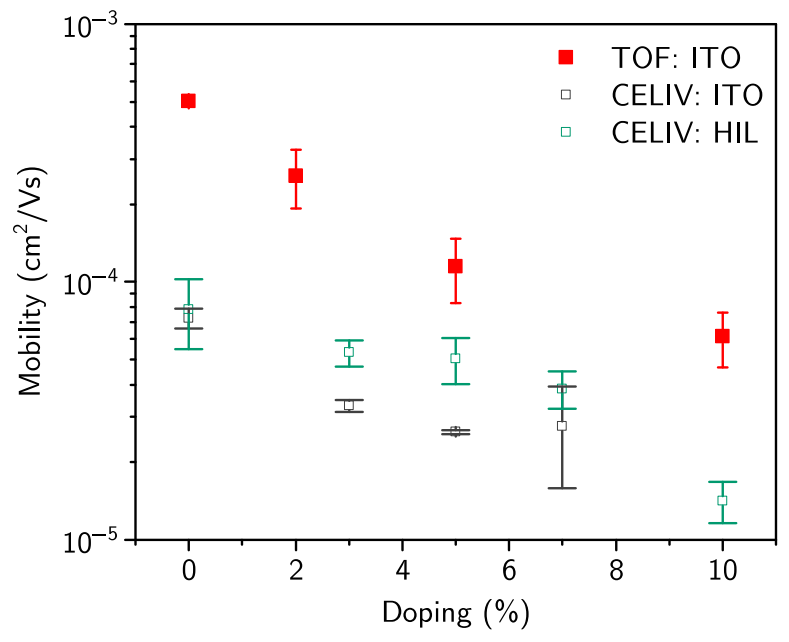

FIG. 6. The absolute mobilities extracted from the CELIV and TOF measurements. Averaging takes place over different samples of the same ratio for CELIV and different fields ranging from $300(\mathrm{~V} / \mathrm{cm})^{1 / 2}$ to $400(\mathrm{~V} / \mathrm{cm})^{1 / 2}$ for TOF.

Although it is well established that CELIV extracts carrier mobilities, different pitfalls for determining the exact value are known [12,22]. To gain another view on the change in charge-carrier mobility due to dipolar doping, TOF measurements are conducted on four different samples with doping ratios again ranging from $0 \%$ to $10 \%$. The value of almost $1 \times 10^{-3} \mathrm{~cm}^{2} \mathrm{~V}^{-1} \mathrm{~s}^{-1}$ for undoped NPB is well in the range of what could be expected from the literature [23]. As temperature-dependent TOF is not available to us, no activation energies can be calculated from TOF mobilities. Figure 6 shows the absolute mobilities extracted from CELIV and TOF and dependent on the doping ratio. It is apparent that TOF, compared to CELIV, overestimates the carrier mobility. However, the effect of the doping concentration on the mobility is systematic. Although, due to lacking a temperature sweep, we cannot reproduce the mobility activation from TOF measurements, we note that a change of mobility over one order of magnitude with doping is expected to have an effect on the overall device performance.

To gain further insight into the energetics of the devices, the situation at the substrate-organic interface has to be evaluated using different techniques.

\section{ULTRAVIOLET PHOTOELECTRON SPECTROSCOPY}

For samples fabricated directly on ITO, a comparison with barriers obtained from ultraviolet-photoelectronspectroscopy (UPS) measurements is conducted. Lowenergy UPS (LE-UPS) is measured for samples with $0 \%$, $2 \%, 5 \%$, and $10 \%$ doping, with excitation energies ranging from 4.4 to $21.22 \mathrm{eV}$, to accurately map the energies in the region of the highest occupied molecular orbitals 
(HOMOs) of the mixture for different film thicknesses in 1-nm steps. Figure 7(a) shows the HOMO region for 1$\mathrm{nm}$-thick films on ITO measured at $7.7 \mathrm{eV}$. The injection barriers are determined from the difference between the HOMO onset and the Fermi energy as indicated by the vertical lines. Figure 7(b) shows the development of the UPS spectra with thickness for the different dopings.

The extracted barriers taken from these measurements for all thicknesses and doping ratios are plotted in Fig. 8 together with a simple band diagram. The ITO work function is directly available from the UPS spectra and amounts to roughly $4.8 \mathrm{eV}$. This is approximately $0.2 \mathrm{eV}$ higher than for the ITO substrates used for electrical measurements

\section{(a)}
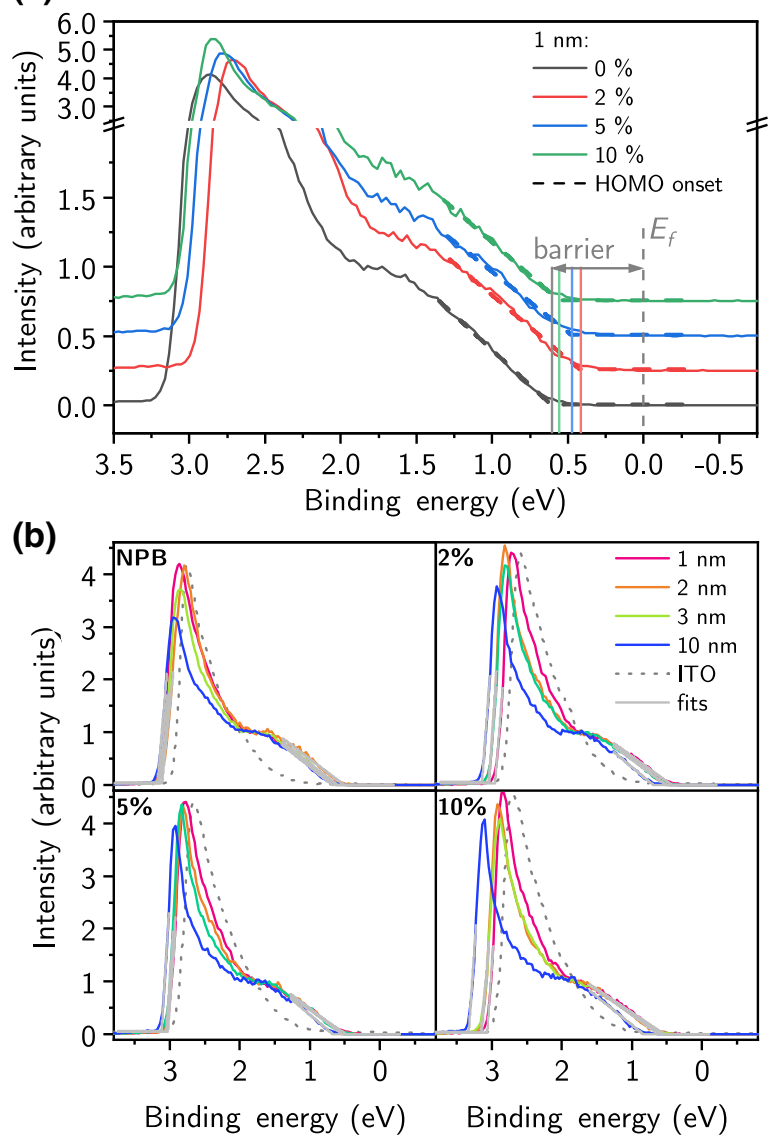

FIG. 7. (a) The normalized UPS spectra of differently doped $\mathrm{NPB}$, measured at an excitation energy of $7.7 \mathrm{eV}$ and a film thickness of $1 \mathrm{~nm}$. The linear fit used to extract the barrier between the HOMO onset and the location of the Fermi level are both shown with dashed lines. The extracted values together with the positions of the secondary electron cut-off (SECO) (fits not shown here) are compiled in Fig. 8. (b) All spectra normalized to the HOMO shoulder of all samples with all thicknesses, measured at $7.7 \mathrm{eV}$ excitation energy. Fits are shown on the left for the SECO (work-function calculation) and on the right for the HOMO onset (barrier). The dashed lines are the measurements on ITO, normalized to the same height. and can be attributed to different manufacturers and sample processing for the substrates, as plasma treatment is impossible in this workflow. From the UPS data, we extract an ionization energy of roughly $5.3 \mathrm{eV}$ for all four doping ratios, which is shown as the dashed line in Fig. 8(a); therefore, the barrier should still be unpinned in all cases[19]. At first sight, the extracted barriers in Fig. 8(b) seem to follow the trend seen in the activation energies extracted from electrical measurements, with a minimum for low-tomoderate doping concentrations. However, this minimum moves to higher ratios with increasing film thickness, while its relative depth is further lowered. As a guide to the eye, the gray arrow in Fig. 8(b) indicates this change. The shift is seen from $2 \%$ for $1 \mathrm{~nm}$ to $5 \%$ at $10 \mathrm{~nm}$, where its depth is only $50 \mathrm{meV}$, compared to $200 \mathrm{meV}$ at $2 \%$.

For further discussion of the UPS data, see the sketch of a band diagram obtained from the measurements in Fig. 8(a). The diagrams can be split into two main features that are evident at a first glance, one being the formation
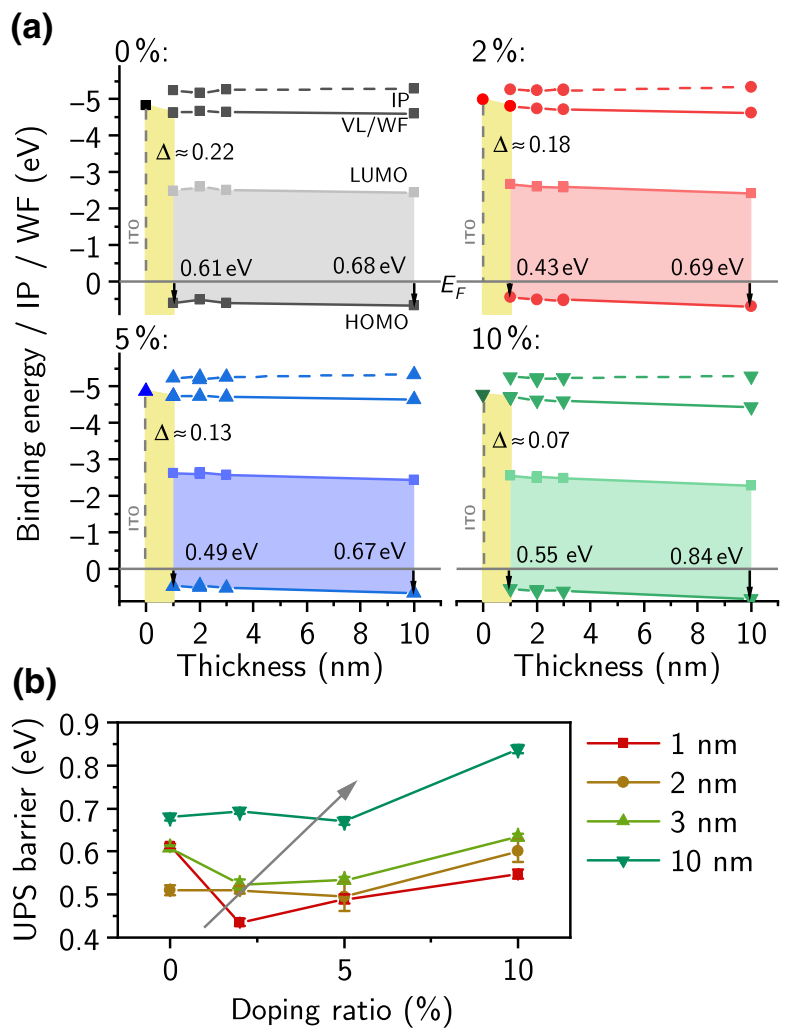

FIG. 8. (a) A simple band diagram extracted from UPS. While the ionization potential is almost unaltered for all samples and thicknesses, the barrier and the vacuum level both shift accordingly. VL, IP, and the HOMO onset are gained from UPS spectra, while the LUMO is calculated by offsetting the HOMO onset by the optical gap as a reference. The energy scale is chosen to be the binding energy relative to the Fermi level, now set to $0 \mathrm{eV}$. (b) The $E_{f}$-HOMO barrier for all thicknesses and doping ratios, plotted against the doping ratio. As a guide to the eye, the shift in the barrier minimum is sketched as a gray array. 
of an interface dipole at the metal-organic interface upon evaporation of the first $1 \mathrm{~nm}$ of organic film. This is common to metal-organic interfaces; it has been reported for NPB [24] as well as $\mathrm{Alq}_{3}$ [25] and will be discussed later on. The second feature is the slight, but steadily shifting, HOMO onset observed without further pronounced features for an increasing film thickness, where both the HOMO onset and the vacuum level or effective work function (solid lines) are shifting toward higher binding energies with the doping ratio, while the ionization potential (dashed line) is almost constant. This band bending can be a result of the dipolar surroundings of NPB when doped with $\mathrm{Alq}_{3}$. A similar dependence has been directly observed and related to the GSP for neat $\mathrm{Alq}_{3}$ layers [25] and has also been seen in molecular-dynamics simulations for neat polar layers by Friederich et al. [26]. With this assumption, the change in vacuum level for the doped layers should be related to the ordered $\mathrm{Alq}_{3}$ dipoles, also leading to the GSP in thicker films. Although the GSP as a macroscopic quantity is typically measured only for thicker layers - e.g., via the Kelvin probe $[9,25]$ - the same shift in energy levels could also be present on the molecular level. With the Kelvin probe, the substrate work function is measured for an increasing thickness of the organic layer and the slope then determines the GSP [25]. The same calculation should be possible with the slope of the vacuum-level change as a change in effective work function. Although the overall layer thickness of only 10 $\mathrm{nm}$ with four points of measurement might not lead to very accurate results, the GSP ranges from between $5 \pm 3$ $\mathrm{mV} / \mathrm{nm}$ for neat NPB to $25 \pm 4 \mathrm{mV} / \mathrm{nm}$ for $10 \%$ of doping. With the Kelvin probe, we measure $0.6 \pm 0.1 \mathrm{mV} / \mathrm{nm}$ for NPB, whereas Noguchi et al. have reported $5 \mathrm{mV} / \mathrm{nm}$ [8]; for a $10 \%$ mixture, we obtain $18 \pm 2 \mathrm{mV} / \mathrm{nm}$ from Kelvin-probe measurements. We therefore assume that the increased band shift for higher doping ratios and the thickness dependence of the extracted barrier are a result of the dipolar order in evaporated polar materials, which also leads to the emerging GSP in doped films.

While the interface dipole leads to a strong shift in vacuum level for neat $\mathrm{NPB}$, for $\mathrm{Alq}_{3}$ doped in NPB the magnitude of the interface dipole is lowered with increased doping. Accompanying the change in the interface dipole, the HOMO onset is lowered by roughly $200 \mathrm{meV}$ directly at the interface, again keeping the ionization potential mostly constant. Although the HOMO levels of NPB and $\mathrm{Alq}_{3}$ are not easily distinguishable, as has also been reported in the past for thin NPB layers deposited on an existing $\mathrm{Alq}_{3}$ layer [27], the relative change of the $\mathrm{HOMO}$ onset is probably not caused by a superposition with $\mathrm{Alq}_{3}$ in the spectra, but by a shift in the NPB HOMO level. This is checked by comparing the shape of the HOMO region in the Hel UPS data (not shown).

As a result, at least two processes determine the extracted UPS barrier: the lowered interface dipole with
$\mathrm{Alq}_{3}$ at the ITO-organic interface and the shift to higher binding energy of the overall HOMO level with an increased density of $\mathrm{Alq}_{3}$ dipoles in NPB. Those two effects counteract each other, leading to a minimum in the extracted barrier. As the interface dipole lowering is an effect bound to the first few monolayers of NPB:Alq 3 on ITO, this effect subsequently loses influence on the observed barrier for thicker layers.

Low-film-thickness measurements, probing the first monolayers of molecules on top of the anode, all show a lower onset or barrier for doped films compared to neat NPB. The barrier for hole injection from ITO into doped NPB could therefore be considered to be lower compared to neat NPB for all of the doping ratios observed here. For charge injection into the bulk, however, it is not the observed HOMO onset but the actual barrier for holes to be injected into layers of NPB some nanometers away from the interface to ITO that is important, as will be discussed below.

\section{DISCUSSION}

The introduction of a polar dopant into the holetransport layer of an OLED lowers the measured activation energies for carrier injection into this polar HTL. It is $a$ priori unclear, however, whether this effect is due to a reduction of the injection barrier at the interface or, as in the case for electrons as measured by Noguchi et al. [6] and described by Kinjo et al. [7] and Altazin et al. [5], is caused by a change in electron affinity and hence carrier densities. In experiment, the effect is dependent on the work function of the substrate and shows a minimum at moderate doping only for low-work-function ITO.

Depending on the work function of the anode or the zero-GSP barrier, two different regimes can be distinguished. For devices where the work function is near the ionization potential of the organic, the effective (injection) barrier for a simple two-layer interface will not reach below a few hundreds of millielectronvolts due to Fermilevel pinning [19]. In this case, a rising doping density and hence an interface charge in NPB is not expected to lower the barrier for hole injection, which is indeed not observed. The influence of the dipolar surroundings of $\mathrm{Alq}_{3}$ in NPB on HIL is, unfortunately, not available for investigation with UPS. Taking the pinned barrier into account, however, again no positive influence is expected.

In the case of low-work-function substrates such as ITO, two processes counteract each other, forming a minimum in the activation energies at about 5\% doping. At first, the presence of $\mathrm{Alq}_{3}$ at the interface lowers the interface dipole at the ITO/NPB interface, accompanied by a change in the energy levels of NPB, shifting the HOMO onset toward lower binding energies for low doping ratios. Upon rising doping, however, the injection barrier into NPB rises as the HOMO onset again shifts to higher binding energies. 
An established theory to describe carrier injection is the Schottky model. Applying the effect of image-charge barrier lowering on thermionic emission, the lowest barrier for carrier injection is not directly at the interface but, rather, a few nanometers away (see the sketch in Fig. 9). The maximum in the potential corresponding to the effective barrier $E_{b \text {,eff }}$ is dependent on the applied electric field (including the GSP by the dipolar dopants), as well as the barrier at the interface in the absence of the field $E_{b, 0}$, i.e., the difference between the Fermi level of the anode and the organic HOMO energy. At first, in the Schottky model, the external electric field causes an effective barrier lowering $\Delta E_{b}$, with

$$
\Delta E_{b}=\sqrt{\frac{e^{3} F}{4 \pi \varepsilon \varepsilon_{0}}},
$$

where $F$ is the external field (for a detailed derivation of the Schottky injection model, see, e.g., Ref. [28]). The maximum in the potential and hence the energetic minimum for carrier injection is field dependent and is given by

$$
x=\sqrt{\frac{\mathrm{e}}{16 \pi \varepsilon \varepsilon_{0} F}} .
$$

With $\varepsilon \approx 3$, this yields a value of roughly $3 \mathrm{~nm}$ for reasonable fields. When the barrier is to be determined from UPS spectra, this effect can thus have an impact on the barrier, especially in the presence of band bending, as is the case here due to the GSP. For amorphous organic materials, surface recombination and hopping across the effective

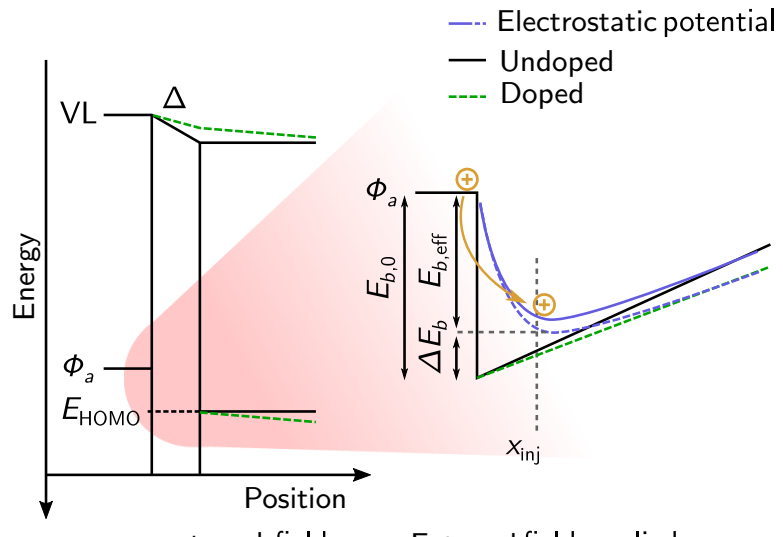

no external field External field applied

FIG. 9. A sketch of the energy levels in the doped and undoped cases. Dipolar doping leads to a reduction in vacuum level and HOMO onset compared to the undoped case. However, the polar species also cause shift and bending of the HOMO in NPB counteracting the field, leading to different injection conditions for both cases. Note that the energy is increasing downward. Left: a general sketch of an energy level without an external field, e.g., in the case of UPS. Right: the levels and electrostatic potentials according to a Richardson-Schottky-type injection with an applied external field. potential barrier a few nanometers away from the interface are also heavily dependent on the carrier mobility. To describe injection of carriers from a metallic contact into an amorphous organic material, Scott and Malliaras have introduced an extension of the classic Schottky model. There, the diffusive motion of carriers within the finite width of the potential barrier is taken into account, leading to mobility-dependent injection [17].

We therefore postulate that neither the change in the barrier nor the change in mobility alone can explain the observed optimum in the current seen in Fig. 2; a superposition of both processes is needed to describe this behavior. The overall scenario in these devices can therefore be summed up as follows: (a) barrier lowering due to a lowered interface dipole in doped films; (b) a subsequent rise in the barrier for higher doping ratios due to different dipolar surroundings and change in the NPB HOMO level; and (c) a decreased hole current at the interface because of diffusion-limited injection in doped NPB with lowered mobility due to dipolar doping.

Additionally, our findings ultimately lead to the conclusion that the dipolar surroundings of $\mathrm{Alq}_{3}$ in NPB change the HOMO energy of NPB, although only low concentrations of $\mathrm{Alq}_{3}$ are used. The energy levels shift with a thickness dependence similar to that observed for films of pure polar material [26].

\section{CONCLUSION}

We show that the introduction of dipolar dopants into the hole-transport layer of an OLED can lower the effective barrier for carrier injection into this polar HTL. The effect is discussed at interface level, as available from electrical and photoemission spectroscopy measurements, and is compared to its counterpart for electron injection on neat $\mathrm{Alq}_{3}$ films, reported previously [7]. As no polar holetransport materials with similar energetic properties are available, a codeposition of two different materials, one being polar, is used instead. Both electrical and photoemission studies of the injection barrier for NPB doped with $\mathrm{Alq}_{3}$ show a minimum in the barrier at roughly 5\% doping. The presence of polar molecules in NPB first affects the interface dipole, leading to lowered hole-injection barriers if they are not pinned, which is the case for ITO but not for HIL. Higher amounts of polar species in the unpolar matrix shift the HOMO of the latter away from the Fermi level, leading to subsequently rising barriers. In addition to the direct influence on the barrier, higher doping ratios negatively affect carrier mobility in NPB, further supporting the optimum at moderate doping. Importantly, dipolar doping allows us to add a tunable GSP to originally unpolar layers in the device stack, directly influencing the charge injection of holes into the device if applied to the HTL. Besides conductivity doping, dipolar doping can hence be a versatile tool with which to tune device performance. 


\section{ACKNOWLEDGMENTS}

This work was supported by a joint project between the German Research Foundation (DFG) and the Swiss National Fonds (SNF) through Grant No. Br 1728/15-1 "CARDYN," as well as by DFG Grant No. Br 1728/201. Further funding was received from the German Ministry for Education and Research (BMBF) through Grant No. 13N13664 "InterPhase" and the Bavarian State Ministry for Science and the Arts through the collaborative research initiative "Solar Technologies Go Hybrid" ("SolTech"). Furthermore, we thank the Japan Society for the Promotion of Science (JSPS) for funding via JSPS KAKENHI (Grants No. JP16H04222 and No. JP18J21921) as well as a JSPS Research Fellowship for Young Scientists (K.S.), the JSPS Summer Program (A.H.), and a BRIDGE Fellowship (W.B.).

[1] T. D. Schmidt, D. S. Setz, M. Flämmich, J. Frischeisen, D. Michaelis, B. C. Krummacher, N. Danz, and W. Brütting, Evidence for non-isotropic emitter orientation in a red phosphorescent organic light-emitting diode and its implications for determining the emitter's radiative quantum efficiency, Appl. Phys. Lett. 99, 163302 (2011).

[2] P. Friederich, R. Coehoorn, and W. Wenzel, Molecular origin of the anisotropic dye orientation in emissive layers of organic light emitting diodes, Chem. Mater. 29, 9528 (2017).

[3] T. Morgenstern, M. Schmid, A. J. L. Hofmann, M. Bierling, L. Jäger, and W. Brütting, Correlating optical and electrical dipole moments to pinpoint phosphorescent dye alignment in organic light-emitting diodes, ACS Appl. Mater. Interfaces 10, 31541 (2018).

[4] W. Brütting, S. Berleb, and A. G. Mückl, Device physics of organic light-emitting diodes based on molecular materials, Org. Electron. 2, 1 (2001).

[5] S. Altazin, S. Züfle, E. Knapp, C. Kirsch, T. Schmidt, L. Jäger, Y. Noguchi, W. Brütting, and B. Ruhstaller, Simulation of OLEDs with a polar electron transport layer, Org. Electron. 39, 244 (2016).

[6] Y. Noguchi, H. Lim, T. Isoshima, E. Ito, M. Hara, W. Won Chin, J. Wook Han, H. Kinjo, Y. Ozawa, Y. Nakayama, and $\mathrm{H}$. Ishii, Influence of the direction of spontaneous orientation polarization on the charge injection properties of organic light-emitting diodes, Appl. Phys. Lett. 102, 203306 (2013).

[7] H. Kinjo, H. Lim, T. Sato, Y. Noguchi, Y. Nakayama, and H. Ishii, Significant relaxation of residual negative carrier in polar $\mathrm{Alq}_{3}$ film directly detected by high-sensitivity photoemission, Appl. Phys. Express 9, 021601 (2016).

[8] Y. Noguchi, Y. Miyazaki, Y. Tanaka, N. Sato, Y. Nakayama, T. D. Schmidt, W. Brütting, and H. Ishii, Charge accumulation at organic semiconductor interfaces due to a permanent dipole moment and its orientational order in bilayer devices, J. Appl. Phys. 111, 114508 (2012).

[9] K. Osada, K. Goushi, H. Kaji, C. Adachi, H. Ishii, and Y. Noguchi, Observation of spontaneous orientation polarization in evaporated films of organic light-emitting diode materials, Org. Electron. 58, 313 (2018).

[10] L. Jäger, T. D. Schmidt, and W. Brütting, Manipulation and control of the interfacial polarization in organic lightemitting diodes by dipolar doping, AIP Adv. 6, 095220 (2016).

[11] P. M. Borsenberger, E. H. Magin, and J. J. Fitzgerald, Hole transport in 1,1-bis((di-4-tolylamino)phenyl)cyclohexane (TAPC) doped poly(styrene)s, J. Phys. Chem. 97, 8250 (1993).

[12] S. Züfle, S. Altazin, A. J. L. Hofmann, L. Jäger, M. T. Neukom, T. D. Schmidt, W. Brütting, and B. Ruhstaller, The use of charge extraction by linearly increasing voltage in polar organic light-emitting diodes, J. Appl. Phys. 121, 175501 (2017).

[13] J. Wagner, M. Gruber, A. Wilke, Y. Tanaka, K. Topczak, A. Steindamm, U. Hörmann, A. Opitz, Y. Nakayama, H. Ishii, J. Pflaum, N. Koch, and W. Brütting, Identification of different origins for s-shaped current voltage characteristics in planar heterojunction organic solar cells, J. Appl. Phys. 111, 054509 (2012).

[14] See www.fluxim.com for "Platform for All-In-One Characterisation of Solar Cells, Fluxim AG" (2017).

[15] T. Sato, H. Kinjo, J. Yamazaki, and H. Ishii, $10^{1} 5 \mathrm{~cm}^{-} 3$ $\mathrm{eV}^{-1}$ level detection of density of states of a p-type polymer by $h v$ dependent high-sensitivity ultraviolet photoemission spectroscopy, Appl. Phys. Express 10, 011602 (2017)

[16] S. M. Park, Y. H. Kim, Y. Yi, H.-Y. Oh, and J. Won Kim, Insertion of an organic interlayer for hole current enhancement in inverted organic light emitting devices, Appl. Phys. Lett. 97, 063308 (2010).

[17] J. C. Scott and G. G. Malliaras, Charge injection and recombination at the metal-organic interface, Chem. Phys. Lett. 299, 115 (1999).

[18] S. Altazin, C. Kirsch, E. Knapp, A. Stous, and B. Ruhstaller, Refined drift-diffusion model for the simulation of charge transport across layer interfaces in organic semiconductor devices, J. Appl. Phys. 124, 135501 (2018).

[19] M. T. Greiner, M. G. Helander, W. M. Tang, Z. B. Wang, J. Qiu, and Z. H. Lu, Universal energy-level alignment of molecules on metal oxides, Nat. Mater. 11, 76 (2012), arXiv:1512.00567. https://doi.org/10.1038/nmat3159).

[20] S. Züfle, S. Altazin, A. J. L. Hofmann, L. Jäger, M. T. Neukom, W. Brütting, and B. Ruhstaller, Determination of charge transport activation energy and injection barrier in organic semiconductor devices, J. Appl. Phys. 122, 115502 (2017).

[21] J. Scherbel, P. H. Nguyen, G. Paasch, W. Brütting, and M. Schwoerer, Temperature dependent broadband impedance spectroscopy on poly- $(p$-phenylenevinylene) light-emitting diodes, J. Appl. Phys. 83, 5045 (1998).

[22] O. J. Sandberg, M. Nyman, S. Dahlström, S. Sandén, B. Törngren, J.-H. Smtt, and R. Österbacka, On the validity of MIS-CELIV for mobility determination in organic thin-film devices, Appl. Phys. Lett. 110, 153504 (2017).

[23] B. Chen, C.-S. Lee, S.-T. Lee, P. Webb, Y.-C. Chan, W. Gambling, H. Tian, and W. Zhu, Improved time-of-flight technique for measuring carrier mobility in thin films of 
organic electroluminescent materials, Jpn. J. Appl. Phys. 39, 1190 (2000).

[24] Q. T. Le, F. Nüesch, L. J. Rothberg, E. W. Forsythe, and Y. Gao, Photoemission study of the interface between phenyl diamine and treated indium-tin-oxide, Appl. Phys. Lett. 75, 1357 (1999).

[25] E. Ito, Y. Washizu, N. Hayashi, H. Ishii, N. Matsuie, K. Tsuboi, Y. Ouchi, Y. Harima, K. Yamashita, and K. Seki, Spontaneous buildup of giant surface potential by vacuum deposition of $\mathrm{Alq}_{3}$ and its removal by visible light irradiation, J. Appl. Phys. 92, 7306 (2002).
[26] P. Friederich, V. Rodin, F. Von Wrochem, and W. Wenzel, Built-in potentials induced by molecular order in amorphous organic thin films, ACS Appl. Mater. Interfaces 10, 1881 (2018).

[27] E. W. Forsythe, V.-E. Choong, T. Q. Le, and Y. Gao, Interface analysis of naphthyl-substituted benzidine derivative and tris-8-(hydroxyquinoline) aluminum using ultraviolet and $\mathrm{x}$-ray photoemission spectroscopy, J. Vac. Sci. Technol. A 17, 3429 (1999).

[28] A. Köhler and H. Bässler, Electronic Processes in Organic Semiconductors (Wiley-VCH Verlag GmbH \& Co. KGaA, Weinheim, Germany, 2015). 